\title{
A dust radiative transfer study of the edge-on spiral galaxy NGC 5908
}

\author{
G. De Geyter, M. Baes, P. Camps, J. Fritz and S. Viaene \\ Sterrenkundig Observatorium, Universiteit Gent, Belgium
}

\begin{abstract}
We present a dust radiative transfer analysis of the edge-on spiral galaxy NGC 5908 . In our previous analysis, it was found that the standard assumption of a double-exponential dust distribution resulted in a poor fit. We investigate the possibility of the dust being distributed in one or more rings. The parameters are constrained using FitSKIRT, a code used to automatically determine the best fitting radiative transfer model given a set of observations. We discuss the possible implications of this dust distribution on the predicted spectral energy distribution.
\end{abstract}

NGC 5908 is an Sb galaxy at a distance $53.4 \mathrm{Mpc}$ and was one of the targets studied in detail in De Geyter et al. (2014). The SDSS $g, r, i$ and $z$-band images were used as input for a FitSKIRT (De Geyter et al. 2013) radiative transfer model in which the dust was distributed in a standard double-exponential disk. Large scale structures showed up in the residual maps, indicating that this model is a poor representation for the actual distribution of dust in NGC 5908. These features had clear ring-like structures, a feature which is not uncommon in galaxies, e.g. the Sombrero galaxy. We find that a model using two rings, one ring operating as an extended disk with a central cavity and another, narrower, ring describing the overdensity, significantly increases the quality of the fits (see the left-hand panel of Fig. 1).

These models are used as an input for a panchromatic radiative transfer simulation, in which we take both evolved and young stellar populations into account. As can be seen from the right-hand panel of Fig. 1, the model with a double-exponential disk and the two-rings model result in very similar SEDs and both fail to reproduce the observed far-infrared fluxes. Therefore, misclassification of dust rings as dust disk seems to have no significant effect on the predicted far-infrared emission and can not even partially explain the far-infrared deficit found in many dust energy balance studies.

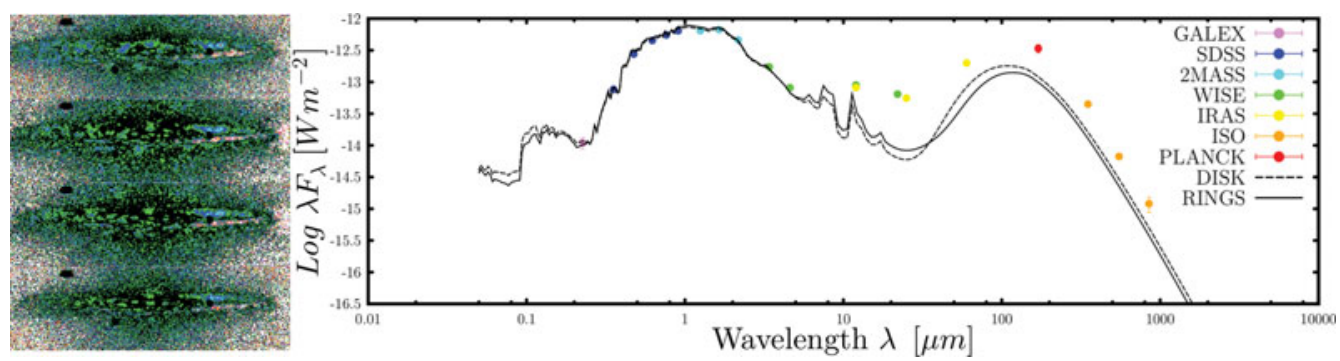

Figure 1. Left-hand panel: The residuals between observations and the model using two rings to describe the dust geometry in NGC 5908. Right-hand panel: The observed fluxes and SED obtained for the models using a disk and two rings with and without a younger stellar component.

\section{References}

De Geyter, G., Baes, M., Fritz, J., \& Camps, P. 2013, A\& A, 550, A74

De Geyter, G., Baes, M., Camps, P., et al. 2014, MNRAS, 441, 869 